\title{
Mastoid Process
}

National Cancer Institute

\section{Source}

National Cancer Institute. Mastoid Process. NCI Thesaurus. Code C12503.

A honeycombed section of bone located near the base of the skull, protruding behind the outer ear. It is connected to the middle ear. 